\title{
A Comparison of Speaking Up Behavior during Conflict with Real and Virtual Humans
}

\begin{abstract}
Breakdowns in team communication are a common source of error. Unfortunately, even when errors are identified, team members may not speak up about the error, often out of fear of confrontation. We propose that virtual humans may be used to help prepare people to speak up. To this end, we conducted a between-subjects study examining speaking up behavior with real and virtual humans. Forty-eight nurses participated in a team training exercise that gave them an opportunity to speak up to a surgeon (either virtual or human, depending on condition) in order to protect a patient's safety. Our results suggest that speaking up behavior with the virtual surgeon closely approximated behavior with the human surgeon: no significant differences were found in participants' use of influence tactics $(p>0.149)$ or in the outcomes obtained ( $p=0.788)$. However, participants were significantly more likely to ask the virtual anesthesiologist for input when working with the human surgeon $(p<0.05)$. Our findings suggest that participants found speaking up to the real and virtual surgeon to be of comparable difficulty. This is an important prerequisite before virtual humans can be used to prepare people to speak up about errors.
\end{abstract}

Keywords: virtual humans, conflict, team training, user study

\section{Introduction}

Teamwork, collaboration, and communication all play an important role in reducing error and improving safety within an organization [1, 2]. Wrong site surgeries are a good example of how breakdowns in communication can lead 5 to serious errors. Wrong site surgery complicates 1 out of 27,686 to 1 out

Preprint submitted to Computers in Human Behavior

May 4, 2015 
of 112,994 surgeries [3, 4]. While wrong site surgeries are rare complications, such a preventable error should never occur. The Joint Commission reports that the most frequent causes of wrong site surgery are communication failures (70\%), procedural noncompliance (64\%) and leadership (46\%) [5. Each of these 10 causes can be addressed by members of the surgical team "speaking up" about safety lapses and errors. Nurses, who fulfill an important role as patient safety advocates, are uniquely positioned to identify and speak up about a wide range of patient safety risks [6]. Unfortunately, several barriers can make it difficult for nurses to speak up, including avoidance of confrontation, accommodation, hierarchy and leadership characteristics [7, 8, 9].

Virtual human-based skill trainers have been developed to teach various interpersonal skills, including conducting medical interviews [10], educating students with autism [11, performing physical exams [12], practicing non-team negotiation skills [13, and coping with bullying [14]. We propose that virtual humans can also be used to develop speaking up trainers, and that these trainers may be able to reduce patient harm caused by interpersonal breakdowns. As a first step toward the goal of using virtual humans to teach health care workers to speak up about errors, we present here the results of a study comparing speaking up behavior with a virtual human to speaking up behavior with a real ${ }_{25}$ human. The goal of this research is to identify whether health care workers employ the same strategies during conflict with real and virtual humans, and to determine whether virtual humans are substantially easier to speak up to, compared to real humans.

In this paper, we explore how actual operating room (OR) nurses reacted when a surgeon (who was either real or virtual, depending on the condition) proposed a course of action that might have endangered a patient's safety. We analyze behavior in terms of the influence tactics participants used when attempting to convince the surgeon to take a safer course of action and in terms of the outcomes participants reached when it became clear that the surgeon could not be convinced to take a safer course of action. We also looked at interactions with the team's anesthesiologist, who was always played by a virtual human, 
and we discuss how participants perceived their virtual teammates, as assessed by a debriefing after the experiment. Our results suggest that behavior with the virtual surgeon closely approximated behavior with the human surgeon, though

40 participants were less likely to involve the virtual anesthesiologist in the decision when working with the virtual surgeon.

\subsection{Types of Virtual Humans}

Virtual humans are computer-generated characters that are visually similar to humans, exhibit human-like behavior, and communicate via natural language

45 and/or gesture. This definition encapsulates the two main continuums of interest when considering a virtual human: its visual realism [15] and its behavioral realism [16]. Visual realism can be influenced by a number of factors, including rendering style [15], animation quality [17, display characteristics (e.g. resolution, size) [18, and display modality (e.g. monitor, head-mounted display, CAVE) [19. Behavioral realism can also be influenced by a number of factors, including behavioral appropriateness [20, behavioral accuracy [21], voice quality [22], non-verbal behavior (e.g. nodding, gestures) [16], gaze behavior [23], and facial/emotional expressions [24].

Both visual realism and behavioral realism can affect how people respond to virtual humans. Kotranza et al. found that increasing a virtual human's visual realism increased participants' acceptance of the virtual human [25]. Putten et al. found that increasing a virtual human's behavioral realism increased feelings of social presence [26] and the amount of words used while speaking with the virtual human [27]. However, while virtual humans with higher levels of visual and behavioral realism are generally perceived more favorably, some exceptions have been found, particularly with regard to visual realism. Nowak et al. found that agents with low anthropomorphism (a highly stylized cartoon face) evoked stronger feelings of social presence than high anthropomorphism agents 28]. McDonnell et al. found that participants rated cartoon faces with lower realism as more appealing and more friendly than slightly more realistic faces [15]; however, highly realistic faces were rated as similarly appealing and 
friendly. Ring et al. found an interaction effect between task domain (medicine vs. social dialog) and visual realism on how an agent was perceived 29]; less realistic virtual humans were perceived as more likable in the social domain, but not in the medical. More realistic virtual humans were perceived as more appropriate in the medical domain, but not the social domain.

When seeking to apply the results of a study involving virtual humans, it is important to consider whether the specific type of virtual human that was studied is of similar visual and behavioral realism to other implementations of virtual humans. If it is not, the research findings may not apply to other types of virtual humans, an important consideration when interpreting and applying the results of this research. The virtual humans used in this research, which had relatively high visual and behavioral realism, are discussed in detail in Section 2.4

\subsection{Realistic Behavior with Virtual Humans}

The Computers as Social Actors theory, also referred to as the Media Equation, states that human interactions with computers and technology are governed by the same rules governing social interactions with humans [30]. Because of this, people often behave as if a computer is a real social agent, even Examples of the Media Equation in practice include that people tend to prefer computers that are similar to themselves [31, display gender biases towards computers that speak in male or female voices [32, and display in-group and out-group biases towards computers based on team identifiers 33 .

The Media Equation phenomenon has also been observed during interactions with virtual humans. Rossen et al. found that users with real-world racial biases exhibited these biases during interactions with a dark skin-toned virtual patient 34. Pertaub et al. observed that negative virtual audiences evoked anxiety in participants practicing public speaking [35. Bailenson et al. found that participants maintained typical interpersonal distances when interacting with a virtual human, and that participants disliked virtual humans who violated their 
personal space [20]. Kotranza et al. found that students expressed empathy towards a mixed-reality human $(\mathrm{MRH})$ when practicing breast exams 25]. Kotranza also compared students' interactions with the MRH to their interactions with a human standardized patient (an actor who helps to train medical students), and found that students used comforting and reassuring touches with similar frequencies for both the MRH and the standardized patient.

While most research has supported the claims of the Media Equation, some research has identified differences in behavior with real and virtual humans. Raij compared medical interviews with a virtual patient to interviews with a human standardized patient [36]. He found that participants' interviews with a virtual patient were less structured and their expressions of empathy were less sincere than those conducted with a standardized patient. Lucas compared self-disclosure behavior when talking with a virtual human and an avatar controlled by another human. Participants reported less fear of self-disclosure, were less likely to engage in impression management behaviors, and engaged in more open behavior when talking with a virtual human compared to a human avatar [37. These differences underscore the importance of examining whether human behavior with specific types of virtual humans mirrors real-world behavior before attempting to use these same types of virtual humans in a new training paradigm.

\subsection{Behavior during Conflict in the Real World}

Behavior during conflict is often described in terms of influence tactics, in which techniques are used to influence others to get what one wants [38. Kipnis identified eight influence tactics: assertiveness, ingratiation, sanctions, rationality, exchange, upward appeal, blocking, and coalition [38. Influence tactics can be divided into hard, assertive approaches (e.g. assertiveness, sanctions, upward appeal) and softer, less forceful approaches (e.g. rationality, ingratiation, exchange) [39]. Harder tactics usually place more strain on relationships and are typically used less frequently than softer tactics, especially when future interactions between the participants are expected [40. Hard influence tactics 
are also used more frequently in online interactions (e.g. virtual teams) than in face-to-face interactions, potentially because people feel they need to exert more influence to be noticed in lower-visibility online mediums 39. Influence tactic usage can be influenced by individual differences, such as feelings of power, trust, and locus of control, and the direction of the influence (e.g. upward, laterally, or downward within an organization) [41. For instance, Kipnis found that sanctions and blocking are rarely used when a subordinate attempts to influence a superior 38 .

Influence tactics research frequently focuses on past use of influence tactics, as assessed through surveys and interviews. Kipnis' original research developed a survey based on 165 essays written by managers describing a past incident where they exerted influence on someone within their organization. He then extracted the original eight influence tactics using a factor analysis of this survey 38 . Yukl later developed a new survey that sought to address limitations in Kipnis' original research [42. More recently, Steizel and Rimbau-Gilabert explored upward influence tactic usage through semi-structured interviews [43]. Wadsworth and Blanchard similarly employed interviews to explore how influence tactic usage differed in virtual teams (e.g. humans working together remotely) and teams that operate face-to-face [39]. Other researchers have directly explored influence tactic usage in laboratory settings. Tjosvold explored the role of cooperative/competitive social contexts on soft and hard tactic usage in a simulated negotiation [44. Van Knippenberg examined the role that potential future interactions had on hard and soft influence tactic usage during task performance 40 .

In this paper, we examine participants' behavior for six of the eight tactics originally described by Kipnis. We chose to exclude sanctions and blocking from our analysis because these tactics are rarely used in upward influence attempts, like that explored in this paper [45, 46] Our examination of influence tactics usage relied on coding of actual participant behavior during a study rather than surveys and interviews, as our participants did not have prior experience working with virtual humans. 


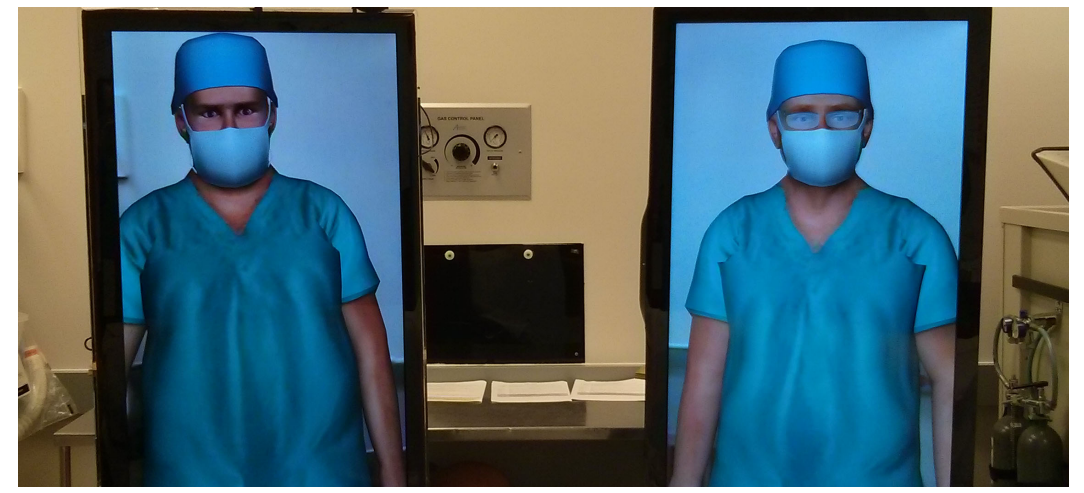

(a) The condition with the Virtual Surgeon and the Virtual Anesthesiologist.

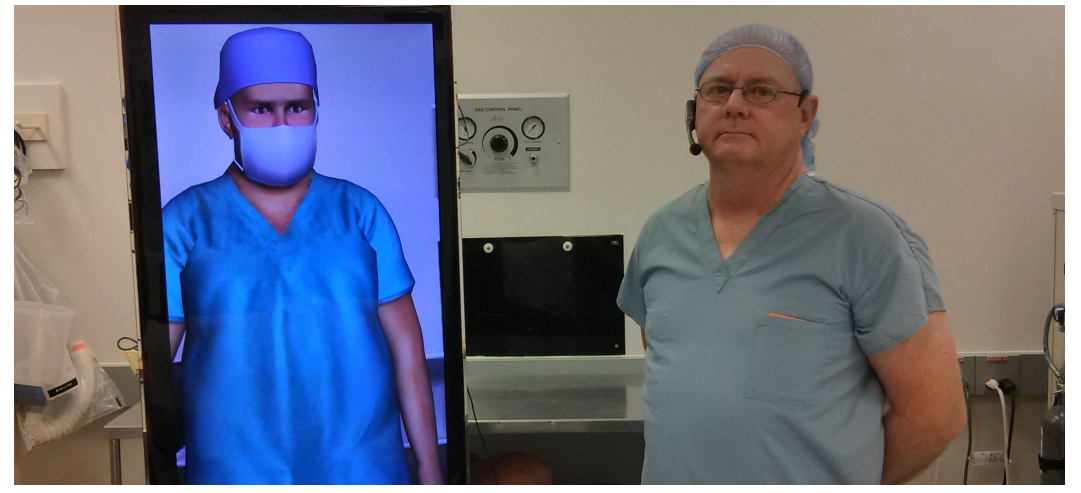

(b) The condition with the Human Surgeon and the Virtual Anesthesiologist.

Figure 1: The two teams used in this study. Human nurses either worked with a human surgeon or a virtual surgeon. The anesthesiologist was virtual in both conditions.

\section{Methods}

\subsection{Design}

To evaluate whether nurses speak up to real and virtual surgeons differently, we conducted a between-subjects study containing two conditions. In the first condition, 26 participants worked with a virtual surgeon and a virtual anesthesiologist. In the second condition, 22 participants worked with a human confederate acting as a surgeon and a virtual anesthesiologist. These two conditions are shown in Figure 1. 


\subsection{Participants}

Participants were nurses working in a hospital operating room located in the south-eastern United States (37 females, 11 males). Ages ranged from 24 to 68 years, with an average age of 44 years. Participants had worked as a nurse

worked as a nurse in the OR for between 0.333 and 43 years, with an average of 10.5 years. Of the 48 participants, 35 reported their race as White, 7 as Asian, and 5 as Black. One participant did not report her race. Participation in the training exercise was mandated by nursing management, but participation study received a $\$ 10$ gift card to a local coffee shop. The entire research protocol was approved by the hospital's Institutional Review Board.

\subsection{Conflict with the Team's Surgeon}

We worked with the hospital's nursing management team to develop a scenario that would give nurses an opportunity to speak up to the team's surgeon. During this scenario, the team's surgeon grew impatient and proposed an unsafe course of action that might harm the team's patient. If this were to occur in the real world, nursing management would ask their nurses to speak up to the surgeon to protect the patient's safety. If the nurse could not personally convince the surgeon to change course to protect the patient, he or she should "stop the line" and contact a charge nurse or nursing management to ensure that breach of protocol did not occur and the patient's safety was protected.

During this scenario, participants worked to prepare a patient for surgery with a high risk of blood loss. The unsafe course of action was proposed by the surgeon at the end of the exercise. As the surgeon was getting ready to start the surgery, he learned that the team's anesthesiologist had forgotten to send blood samples to the blood bank for processing, which meant replacement blood was not yet available. After learning this, the surgeon grew angry with the anesthesiologist and ordered him to send the samples immediately. He then 
have time to process the samples before replacement blood was needed. This course of action might endanger the patient's safety, who could be seriously compromised or even die if blood was needed unexpectedly or if the blood bank took longer than expected to process the samples, which could occur if

the patient had antibodies. In addition to being unsafe, the surgeon's proposed course of action was also in direct violation of hospital policy, which requires two blood samples to be sent and processed before beginning surgery. The nursing management team was categorical that all nurses should (a) recognize that the surgeon's proposed course of action could potentially endanger the patient and (b) speak up and call the charge nurse, if needed, to protect the patient.

\subsection{Virtual Team Members}

The virtual surgeon and virtual anesthesiologist are shown in Figure 2. The virtual humans used in this study had relatively high visual and behavioral realism. They were life-size and interacted with participants using speech and gesture. The virtual humans' speeches were prerecorded by voice actors, and gestures were created using motion capture. The virtual humans were displayed using high-physicality interaction modules, as described by Chuah [47]. The virtual humans were rendered life-size on 40" televisions and could track participants' positions using a Microsoft Kinect, which allowed them to make eye contact with participants. Perspective-correct rendering was used to create an illusion of depth and see-through backgrounds were simulated using pre-captured panoramic images of the environment; the use of perspective-correct rendering also allowed the see-through background to adjust based on participants' position in the room. The virtual humans' head gaze was controlled through a simple Markov model; the virtual humans looked at whoever was speaking, but could randomly glance at the other team members. They also blinked and mimicked idle motions when not speaking - these idle animations were created using motion capture. To avoid introducing potential gender and racial confounds, both the virtual surgeon and the virtual anesthesiologist were modeled as average Caucasian males. This combination of race and gender is representative of 
the majority of surgeons and anesthesiologists practicing in the United States [4].

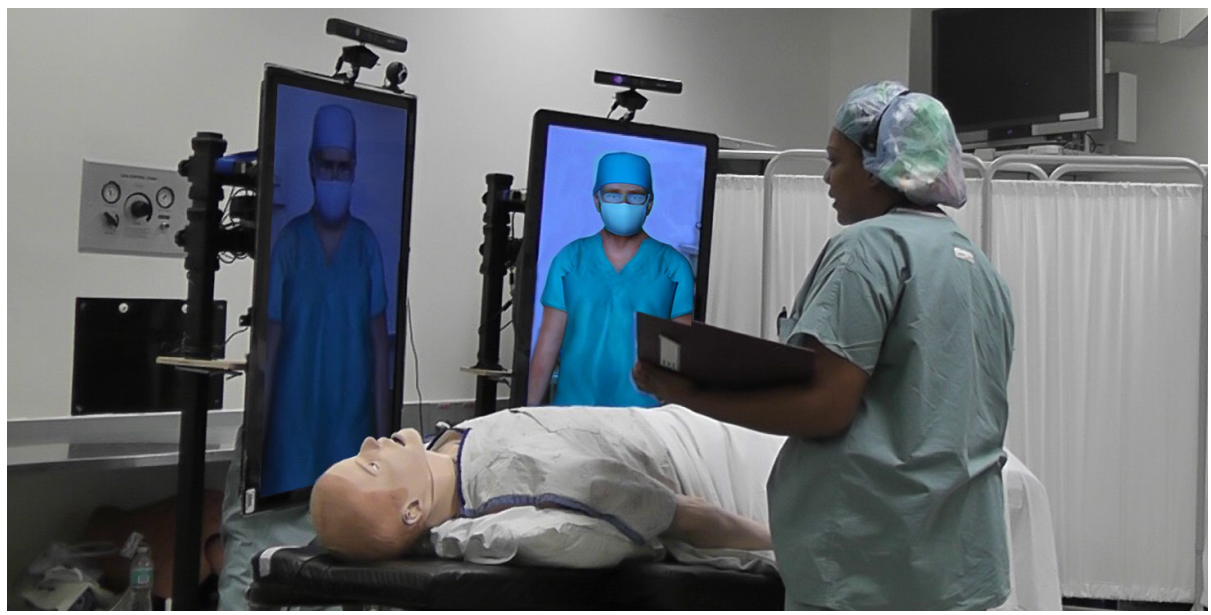

Figure 2: A participant working with the virtual anesthesiologist and the virtual surgeon, who are standing behind the mannequin patient simulator. The study took place in a former operating room that has been converted to a simulation lab.

The team's patient in this exercise was a mannequin simulator (see Figure 2. The scenario called for a patient who was developmentally-delayed and nonverbal, so that the patient did not interact with the team. The mannequin simulator blinked, breathed, and generated normal vital signs, which were displayed on a nearby monitor. The patient was undergoing a high-risk scoliosis surgery associated with considerable blood loss.

The virtual humans were controlled by a Wizard-of-Oz (WoZ). Humanfactors researchers frequently use WoZs to reduce confounding effects that can be introduced by speech recognition errors or speech understanding errors [12, 49, 50. The wizard controlled the virtual surgeon and the virtual anesthesiologist simultaneously, using an interface that allowed him to trigger the virtual humans' speeches using pre-specified lists. This interface was organized by character and topic, to allow for rapid selection. The interface also intelligently suggested responses based on the previous action performed. The wizard followed a specific script for each stage of the interaction, but made adjustments when 
participants behaved unexpectedly. The virtual humans were capable of making nine generic statements, such as "Yes", "No" , "OK", and "I'm not sure", which allowed the wizard to respond to unexpected questions or statements. In order to create a consistent experience for each participant, the same wizard was used during the entire study.

\subsection{Human Confederate}

Figure 1 shows the two conditions used in this study. Once a human confederate had been selected, the virtual surgeon was modified to resemble the human confederate in terms of height, body type, skin coloration, and facial appearance. As the human confederate wore glasses, glasses were also added to the virtual surgeon. To ensure that human surgeon and the virtual surgeon sounded similar, the human confederate recorded the audio used by the virtual surgeon.

The confederate was a trained standardized patient who regularly assists with medical student training exercises. Standardized patients are human actors commonly used to train medical students to practice medical interviewing and physical examinations. As such, they are trained to portray specific individuals and exhibit specific medical complaints or symptoms. While standardized patients do not normally play the role medical professionals, they are familiar with the medical domain and understand that it is important to behave consistently during each training session. The confederate was recruited from the pool of available standardized patients and was paid the standard rate of $\$ 20$ 265 per hour during the study.

The confederate received training on the script from the WoZ operator who controlled the virtual humans during the study. He explained the scenario and demonstrated a standard interaction where the surgeon and the anesthesiologist were both virtual, and then had the confederate practice playing his role until he was comfortable with it. After this training session, the confederate took home paper scripts for further study and familiarization. These paper scripts were also used during the study as a guide to help the confederate perform 
consistently during the exercise.

Mostly, the human confederate successfully mimicked the behavior of the

virtual surgeon. One notable exception to this was the human confederate's gaze behavior. The human confederate made significantly less eye contact with participants, as he frequently consulted his script during the interaction. He also occasionally responded incorrectly to a participant's statement or ad-libbed an unscripted response. The implications of these variances will be considered further in the Limitations section.

\subsection{Procedure}

Upon arrival, the participant was he or she would be working with a team to prepare a simulated patient for surgery, and would be playing the role of a circulating nurse. Participants were instructed to treat their virtual team members exactly like they would treat real humans. They were also told that their behavior during the exercise would remain completely confidential, and that the exercise was not being used as a performance assessment. Participants were not warned that the surgeon would behave in a manner that could potentially endanger the patient's safety. Participants also completed a brief tutorial that allowed them to practice speaking with a virtual human.

Stage 1: The Briefing. The exercise consisted of two stages: the briefing stage and the timeout stage. In the briefing stage, participants worked with a surgeon and a virtual anesthesiologist to ensure that the patient was ready for the start of anesthesia. The surgeon guided this stage, working through the actual checklist used in this hospital's operating rooms. The surgeon asked questions of the participant and the virtual anesthesiologist as needed. The virtual anesthesiologist occasionally interrupted the surgeon to ask a question. Participants could also interrupt with questions or comments. At the end of the briefing, the surgeon learned that blood samples had not been drawn and instructed the virtual anesthesiologist to draw the blood after inducing the patient. 
Stage 2: The Timeout. After the surgeon completed the checklist, participants moved on to the timeout stage. In this stage, participants worked with the surgeon and the virtual anesthesiologist to confirm that the patient was ready for the incision that would begin the surgery. The surgeon guided this stage, working through a second, shorter checklist. After asking the participant several basic questions, the surgeon asked the virtual anesthesiologist if the blood was now available. The virtual anesthesiologist reported that he had forgotten to send the blood to the lab. This angered the surgeon, who berated the virtual anesthesiologist and then announced that, because they were running late, the team needed to send the blood samples immediately and start the surgery without waiting for the results. This proposed course of action could potentially endanger the patient's safety, because blood might be needed sooner than expected, and because the patient's blood could have antibodies that would slow the blood preparation processes. This proposed course of action was also in violation of hospital policy, which states that two samples must be sent to the blood bank before the incision can be made.

The Speaking Up Moment. If participants spoke up to the surgeon and expressed concerns about the surgeon's proposed course of action, he would repeatedly object to and dismiss their concerns. His objections were that the patient was unlikely to have antibodies because he had never been transfused before, that there was sufficient time to get blood before it would be needed, and that waiting could harm the patient because of the additional time he would be under anesthesia (these objections were developed in collaboration with nursing management and an anesthesiologist). After a participant spoke up three times, the scenario called for the surgeon to announce that he was not going to listen to the participant anymore and that he was going to begin the surgery. At this point participants could either back down or "stop the line" and call a supervisor. The anesthesiologist did not say anything during the speaking up moment, unless he was asked a question by the participant. If asked to intervene, he was programmed to say that he was not going to get involved and would let 
the surgeon and the nurse make the decision.

\subsection{Metrics}

Participants' speaking up behavior with the surgeon was assessed through post-hoc video and transcript coding. Borrowing from Gorden's Coding Interand coded the outcome of his or her conflict with the surgeon. Videos of participants' interactions were consulted when the transcripts were unclear. After finishing the initial coding, the two authors discussed their observations and, following negotiation, agreed upon the five outcomes shown in Table 2 They ond round of coding, differences were minor and agreement was quickly reached across all study participants.

Upon finalizing the coding of the conflict outcomes, the same two authors then analyzed and coded the language participants used during conflict, using reported in Table 1 1 . They then analyzed and re-coded the transcripts using these updated definitions. After the second round of coding, differences were minor and agreement was quickly reached across all study participants.

\section{Results and Discussion}

In this section, we examine the influence tactics participants used with the surgeon, how they resolved the conflict, and their interactions with the virtual anesthesiologist during the speaking up moment. We also discuss participants' reactions to the virtual humans and the training exercise as a whole. Because 


\section{Influence Tactic Definition}

\begin{tabular}{ll}
\hline Rationality & Attempts to use logic or reason to persuade the surgeon \\
\hline Assertiveness & $\begin{array}{l}\text { Challenges the surgeon using references to hospital pro- } \\
\text { cedure, force of will, or statements not involving reason } \\
\text { or inference. }\end{array}$ \\
\hline Ingratiation & $\begin{array}{l}\text { Attempts to use empathy, appeasement, or deference } \\
\text { to persuade the surgeon }\end{array}$ \\
\hline Upward Appeal & Asks an authority figure to intervene on their behalf \\
\hline Coalitions & Asks someone who is not an authority figure to support \\
their position
\end{tabular}

Table 1: These definitions were extracted from the questions used in Kipnis' original survey and then adapted to fit the population of this study.

the conditions contained a different number of participants, behavioral results are reported as percentages rather than counts.

\subsection{Influence Tactic Usage During Conflict}

Of our 48 participants, only 25 (52\%) attempted to influence the surgeon; the remaining 23 either deferred to the surgeon or actively supported his proposal. As such, these 23 (48\%) participants used no influence tactics and were thus excluded from this portion of our analysis. Of the remaining 25 participants, 15 worked with the virtual surgeon and 10 worked with the human surgeon. A chi-square test revealed no significant differences between conditions in the number of participants who attempted to influence the surgeon $(p=0.398)$.

Figure 3 reports the percentage of participants who used each tactic at least one time. Participants frequently used more than one influence tactic, which meant that influence tactic usage could not be assessed using a MANOVA, as the independence assumption was violated. Instead, individual t-tests were con- 
ducted for each of the six influence tactics. The coalition tactic was used more often $(p<0.05)$ with the human surgeon than the virtual surgeon. However, no significant differences were found between conditions for the remaining five influence tactics $(p>0.149)$.

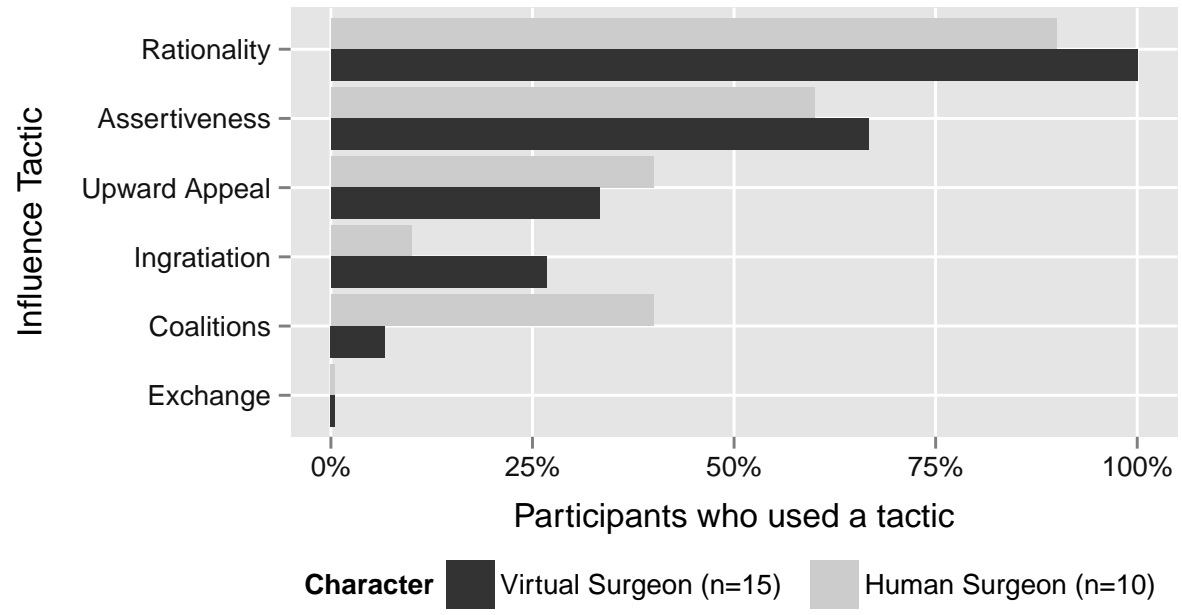

Figure 3: The percentage of participants who employed a specific tactic. No participants used exchange.

Figure 4 reports the number of different tactics participants used (e.g. using rationality and assertiveness). Figure 5 reports the total number of times participants attempted to influence the surgeon (e.g. using rationality three times and assertiveness twice). No significant differences were observed in the number of different tactics used between the two conditions $\left(p=1.000, \mu_{\text {Virtual }}=\right.$ $\left.2.4, \mu_{\text {Human }}=2.4\right)$. Similarly, no significant differences were observed in the total number of times participants attempted to influence the surgeon ( $p=$ $\left.0.292, \mu_{\text {Virtual }}=4.87, \mu_{\text {Human }}=3.90\right)$.

Figure6 6reports how much time participants spent talking during the speaking up moment (this time does not include time when the surgeon spoke or periods of silence). No significant differences were observed in how much time participants spent talking to the human or virtual surgeon during the speaking up moment $\left(p=0.788, \mu_{\text {Virtual }}=29.01\right.$ seconds,$\mu_{\text {Human }}=27.45$ seconds $)$. 


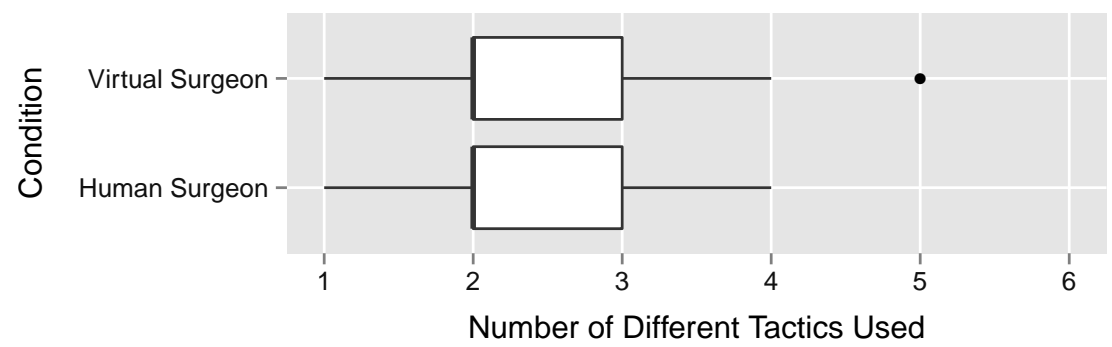

Figure 4: This boxplot reports how many of the six different tactics were used by participants. The dot indicates one outlier who employed five of the six tactics.

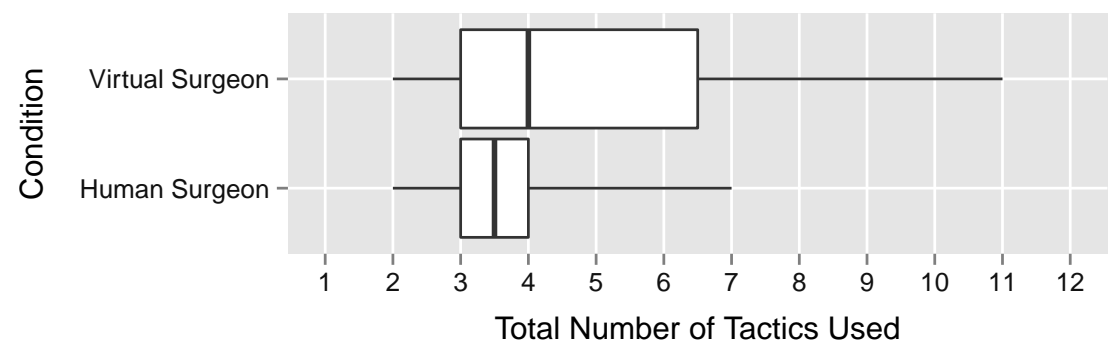

Figure 5: This boxplot reports the total number of times participants attempted to influence the surgeon.

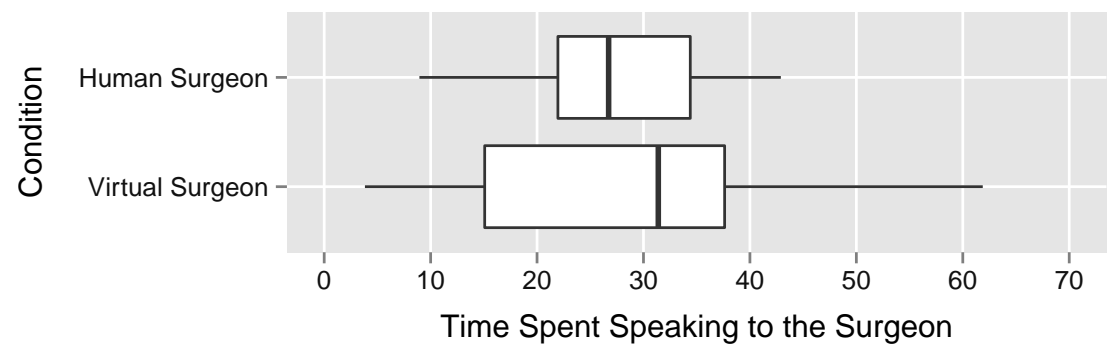

Figure 6: The time reported in this boxplot is when participants were speaking. It does not include when the surgeon spoke or periods of silence.

\subsubsection{Discussion of Influence Tactics Usage During Conflict}

With the exception of the coalition tactic (which will be explored further in Section 3.3.), these results suggest that participants' attempts to stop the surgeon from beginning the surgery were not significantly influenced by whether the surgeon was real or virtual. Our results show that participants used influence 
tactics similarly in both conditions: they used influence tactics at the same rates, used a similar range of tactics, made a similar number of attempts to stop the surgeon, and spent similar amounts of time negotiating regardless of whether the surgeon was real or virtual. While these results cannot demonstrate statistical equivalence between behavior with real and virtual surgeons, they do suggest that whatever differences may exist are minor and unlikely to hinder virtual humans with high visual and behavioral realism from being used for speaking up training.

Several researchers have found that rationality is one of the most frequently used influence tactics 38, 42. This finding was confirmed in our results for both the virtual and human surgeon. Twenty-four out of twenty-five participants used rationality when attempting to persuade the surgeon to wait to begin the surgery. Surprisingly, assertiveness was also frequently used by participants, despite results in the literature suggesting that assertiveness is not commonly used when attempting to influence a superior [38. The high incidence of assertiveness during this study may be due to the surgeon's unwillingness to be convinced by reason; of the 14 participants who employed both rationality and assertiveness, 9 of them resorted to assertiveness only after first attempting to use rationality to convince the surgeon to wait to begin the surgery. Participants may also have been more willing to use assertiveness because of the high stakes nature of the scenario. Most influence tactics research has been conducted in business settings, where lives are rarely, if ever, at stake. Participants may have been more willing to use assertiveness because of the threat to their simulated patient's safety.

\subsection{Outcomes to the Speaking Up Moment}

Next we examine the outcome participants reached during the speaking up moment. Outcomes describe the final outcome to the exchange between the surgeon and the nurse, as distinguished from the tactics used while attempting to influence the surgeon. The five outcomes we identified are summarized in Table 2. These five outcomes were identified by two of the authors through 
a post-hoc video review and analysis of each participant's behavior during the speaking up moment, as described in Section 2.7. The 23 participants who were excluded from the influence tactic analysis for failing to speak up to the surgeon achieved the "No objections" outcome. One participant's data was excluded because the human surgeon mistakenly agreed to delay the surgery until blood was available.

\section{Outcome Description}

\begin{tabular}{ll}
\hline Stopped the line & Refused to let the surgeon begin the operation \\
\hline Incident report & Agreed to proceed, but filed a report on the incident \\
\hline Shifted responsibility & Voiced concern, but left the decision up to the surgeon \\
\hline Gave in & Initially voiced concern, but later backed down \\
\hline
\end{tabular}

No objections $\quad$ Raised no objections to the surgeon's decision

Table 2: The outcomes are ordered based on the degree of resistance participants offered to the surgeon, in descending order.

Figure 7 shows the percentage of participants in each condition who reached each outcome. Fisher's exact test revealed no significant differences between the two conditions $(p=0.869)$. Of these five outcomes, nursing management considered stopping the line to be the only acceptable outcome, from a patient safety perspective. Only $20 \%$ of participants in both conditions resolved conflict with the surgeon by stopping the line. Another $25 \%$ to $40 \%$ of participants, depending on condition, voiced concern about the surgeon's proposed course of action, but failed to stop the line and allowed the surgeon to begin the surgery. Filing an incident report, which was the most assertive resolution method that 440 still fell short of stopping the line, was observed once with the virtual surgeon, but did not occur with the human surgeon. The remaining $40 \%$ to $55 \%$ of participants, depending on condition, offered no objections to the surgeon's proposed course of action. Of these participants, six discussed the decision with the surgeon without offering objections to it; five of these six were in the human 


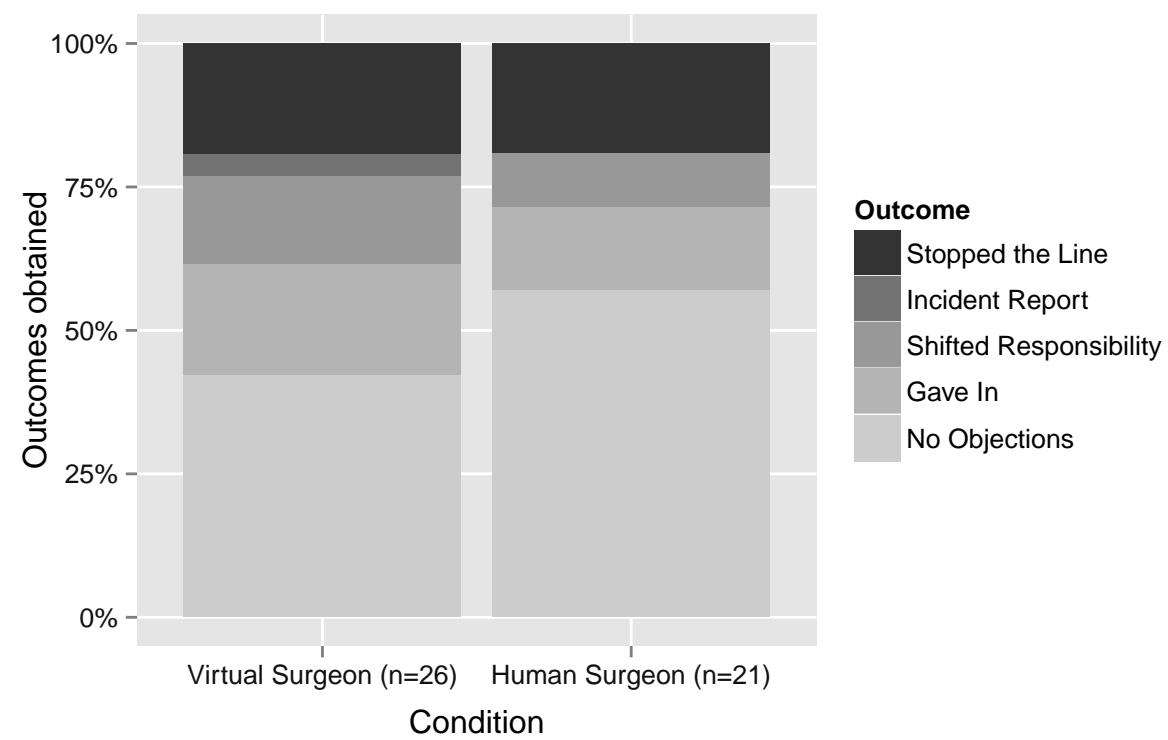

Figure 7: The percentage of participants for each outcome. No participant filed an incident report in the human surgeon condition. Outcomes are ordered based on the amount of resistance presented to the surgeon.

surgeon condition. The remaining participants who offered no objections agreed immediately with the surgeon's decision to proceed.

\subsubsection{Discussion of Outcomes to the Speaking Up Moment}

These results indicate that participants' behavior during the speaking up moment was not substantially affected by whether the surgeon was real or virtual.

450 No significant differences were observed between conditions. Most importantly, the same proportion of participants $(20 \%)$ stopped the line in both conditions; this is critical, as stopping the line was both the most desired behavior and the most difficult behavior. It does appear that participants were more likely to offer no objections to the human surgeon. However, it is difficult to draw conclusions about why participants offered no objections; some participants offered no objections because, based on their previous real world experience, they felt the surgeon's proposed course of action was appropriate. Unfortunately, we 
do not have a way to quantify how many of the participants who offered no objections did so because they genuinely did not object, compared to lacking the confidence to speak up to the surgeon.

Although it may seem surprising that only $20 \%$ of participants stopped the line, there are several factors that can make speaking up as a nurse difficult. Even though speaking up is ideal, the perception of a hierarchical relationship between nurses and surgeons can make speaking up difficult [52]. Nurses sometimes feel that a surgeon's more extensive medical training makes him or her more qualified to make decisions about patient care [53. Participants working with the virtual surgeon mentioned both of these difficulties during the debriefing. Six participants deferred to the virtual surgeon for hierarchical reasons, using phrases like "You're the surgeon, it's up to you". Three participants stated that the virtual surgeon's experience and position made him more qualified to make the decision. From a training perspective, it is encouraging that participants encountered these real-world difficulties when speaking up to the virtual surgeon, as it suggests that virtual humans may also be used to help prepare people to overcome these barriers in the real world.

Filing incident reports and shifting responsibility in a simulation context is especially interesting. Both of these behaviors are examples of self-protective voice [54] and represent attempts to guard oneself from potential repercussions should the surgeon's behavior harm the patient. In both cases, the nurse sought to establish that she had objected to the surgeon's behavior but had been unable to stop him. Observing self-protective behavior in the context of a simulation is important, as participants knew that there would be no real-world repercussions if they failed to protect the simulated patient (they had been informed that this simulation was not an evaluation and that their performance would not be reported to management). Accordingly, the use of self-protective behaviors suggests that these participants automatically engaged in the same behavior they would use during real-world conflict. Observing these behaviors with both the human and the virtual surgeon further strengthens the conclusion that behavior with a virtual surgeon with high visual and behavioral realism closely 
approximates behavior with a human surgeon.

\subsection{Interactions with the Anesthesiologist}

As noted previously, a significant difference was seen in how participants used the coalition tactic with the real and virtual surgeon. This difference can be attributed to a more general difference in how participants interacted with the virtual anesthesiologist. Figure 8 reports the percentage of participants in each condition who asked the virtual anesthesiologist to comment on the surgeon's proposed course of action. Fisher's exact test revealed that participants asked the anesthesiologist to weigh in significantly more often when working with the human surgeon $(p<0.05)$.

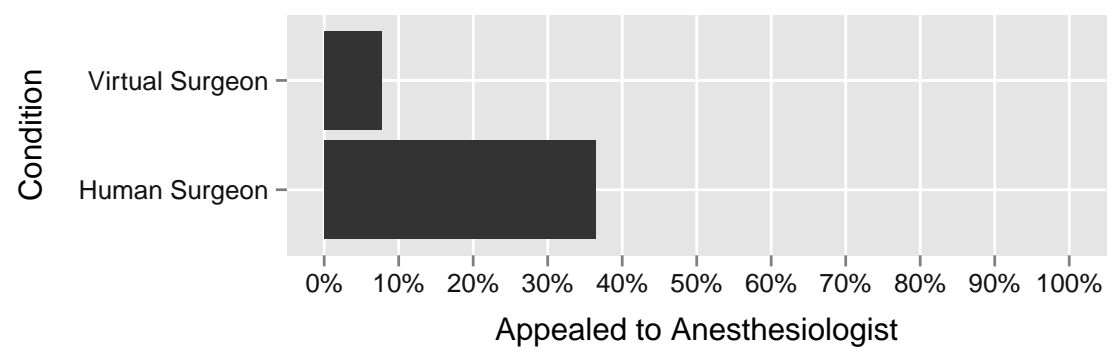

Figure 8: The percentage of participants who asked the anesthesiologist to weigh in on the surgeon's proposed course of action.

Participants asked the anesthesiologist to weigh in for one of two reasons: they either sought to gain the anesthesiologist's help in influencing the surgeon to change course, or they sought confirmation on whether the surgeon's proposed course of action was appropriate. Participants who merely sought confirmation typically agreed to proceed after the anesthesiologist said he was not going to get involved, while participants who were looking for support generally continued to negotiate with the surgeon. One participant who was looking for support went so far as to berate the anesthesiologist for not wanting to get involved, reminding the anesthesiologist that he would be responsible for resuscitating the patient in the event something went wrong. 
There are at least two possible explanations for why participants were more

likely to ask the virtual anesthesiologist for help when working with the human surgeon: either participants felt more threatened by the human surgeon and thus felt a greater need of assistance from the anesthesiologist, or working with the human surgeon caused participants to be more aware of the anesthesiologist as a fellow teammate and thus were more likely to seek his input. It is difficult to judge which explanation is more likely. In the human surgeon condition, none of the participants who asked the anesthesiologist for input stopped the line. This could indicate that these participants were uncomfortable stopping the surgeon themselves and thus asked the anesthesiologist to intervene on their behalf. However, in the debriefings, participants frequently said that working with a human surgeon made them feel like the virtual anesthesiologist was more realistic; most participants attributed this to observing the human surgeon behaved as if the anesthesiologist were a real member of the team. More research is required before any firm conclusions can be drawn. Whatever the reason may be, it is encouraging that participants were willing to ask the virtual anesthesiologist for help. This lends further support to the notion that virtual humans of high visual and behavioral realism can be successfully applied to team training, as it shows that participants viewed the anesthesiologist as a member of the team who could contribute to the decision making process.

\subsection{Impressions about Training with Virtual Humans}

Several participants reported feeling more confident and more motivated to speak up after practicing with the virtual humans. Other participants reported that they did not find the training personally useful because they already felt comfortable speaking up; however, they did feel that it would be useful for less experienced nurses who did not feel empowered to speak up and stop the line. Intriguingly, several of these nurses did not stop the line, indicating that they either were not genuinely completely comfortable speaking up, or failed to identify the patient safety issue. This inconsistency between these participants' self-assessment of their own ability and their actual behavior underscores the 
importance of this type of training.

Participants in both conditions reported that the exercise provided an effective opportunity to practice speaking up to a surgeon. Multiple participants in the virtual surgeon condition reported feeling like they had been speaking up to a real surgeon, commenting on his tone of voice, his anger and impatience, and the arguments he raised. Participants were also positive about the interaction with the entire virtual team, saying that the way the virtual humans looked at participants, interacted with each other, and responded verbally to participants made them feel like they were working with a real team.

Participants who worked with the human surgeon also agreed that the surgeon behaved similarly to some surgeons they had worked with in the past. When asked about the dynamic of working with both the virtual anesthesiologist and the human surgeon, most participants felt that having another human there made the virtual anesthesiologist seem more realistic. These participants frequently attributed this increase in realism to seeing the human surgeon behave as though the virtual anesthesiologist was a real member of the team. However, there were also some participants who felt that the contrast between the human surgeon and the virtual anesthesiologist made the anesthesiologist feel less realistic.

\subsection{Limitations}

There are two important limitations in this study that need to be acknowledged. The first limitation is the gender imbalance inherent in our population. While male nurses are more common than in the past, female nurses remain more common than their male counterparts. This imbalance was reflected in our participants where $77 \%$ were female. This limitation is especially important given that gender effects are not uncommon in social behavior. As such, our results may not generalize to other populations that are more heterogeneous.

The second limitation relates to variability in the behavior of the human and virtual surgeons. While the human surgeon attempted to be consistent in his tone of voice, posture, and phrasing, there was some unavoidable variability 
that is inherent in any interaction involving human confederates, and it is pos-

sible that these variations influenced participant behavior. A more important limitation in the human confederate's behavior was a systematic variation from the virtual human's behavior. The human confederate exhibited different gaze behavior due to his use of a script to stay on track during the exercise. As such, he spent less time looking at the participant than the virtual surgeon, who was programmed to look at whoever was speaking (either the participant or the virtual anesthesiologist). It may be that participants would have been more hesitant to confront the human surgeon if he had maintained more consistent eye contact. However, this type of gaze behavior is not uncommon in the OR, where surgeons and anesthesiologists frequently have to look at patient charts, checklists, or monitors, and thus cannot always maintain eye contact with their teammates. As such, the human surgeon's gaze behavior was unlikely to be interpreted as unrealistic or non-dominant behavior.

\section{Conclusions}

Our results suggest that virtual humans with high visual and behavioral realism can be used for speaking up training. Participants used similar tactics while speaking up with both the real and virtual surgeon, and participants did not find it substantially easier to stop the line with the virtual surgeon. Self-protective behavior was observed with the virtual surgeon, which suggests that participants responded to conflict with the virtual surgeon using the same techniques they use in the real world. These results suggest that speaking up to virtual humans with high visual and behavioral realism can be challenging and approximates the experience of speaking up to a real human. However, it is important to consider that these results may not hold true for virtual humans that have less visual or behavioral realism, or to different domains. Future work will explore whether skills gained while speaking up to virtual humans transfer to conflict in the real world. 


\section{References}

[1] A. Camino, Management of safety, safety culture and self assessment, in: International Conference on Nuclear Energy in Central Europe, 2000, pp. $1-15$.

II

URL http://www.iaea.org/inis/collection/NCLCollectionStore/ _Public/34/087/34087529.pdf

[ [2] R. Helmreich, J. Wilhelm, Outcomes of crew resource management training, The International journal of aviation psychology 1 (4) (1991) 287-300.

口 URL http://www.tandfonline.com/doi/abs/10.1207/ s15327108ijap0104_3

[3] M. R. Kwaan, D. M. Studdert, M. J. Zinner, A. A. Gawande, Incidence, Patterns, and Prevention of Wrong-Site Surgery, Arch Surg (141) (2006) 353-358.

[4] S. C. Seiden, P. Barach, Wrong-Side/Wrong-Site, Wrong-Procedure, and Wrong-Patient Adverse Events: Are they preventable?, Arch Surg (141) (2006) 931-939.

प

URL http://archsurg.jamanetwork.com/article.aspx?articleid= 398954

[5] Sentinel event statistics data: root causes by event type (2014).

URL http://www.jointcommission.org/sentinel_event_statistics

[6] M. M. Sayre, D. McNeese-Smith, L. S. Leach, L. R. Phillips, An Educational Intervention to Increase "Speaking-Up" Behaviors in Nurses and Improve Patient Safety, Journal of Nursing Care Quality 27 (2) (2012) 154-160. doi:10.1097/NCQ.0b013e318241d9ff.

[7] B. S. Eason F, Conflict management: assessing educational needs, Journal for Nurses in Staff Development 15 (3) (1999) 92-96. 
URL http://journals.lww.com/jnsdonline/Abstract/1999/05000/

口 [8] S. J. Cavanagh, The conflict management style of staff nurses and nurse managers., Journal of advanced nursing 16 (1961) (1991) 1254-1260.

URL http://onlinelibrary.wiley.com/doi/10.1111/j.1365-2648. 1991.tb01536.x/abstract

${ }_{630}$ 口 $[9]$ A. Hendrich, E. J. Thomas, C. G. Holzmueller, Teamwork in the Operat-

a ing Room: Frontline Perspectives among Hospitals and Operating Room Personnel, Anesthesiology (105) (2006) 877-884.

URL http://www.ncbi.nlm.nih.gov/pubmed/17065879

[10] A. Wendling, S. Halan, P. Tighe, L. Le, T. Euliano, B. Lok, Virtual Humans

635 ㅁ versus Standardized Patients: Which lead residents to more correct diagnoses?, Academic Medicine 86 (3) (2011) 384-388. arXiv:NIHMS150003, doi:10.1016/j.biotechadv.2011.08.021.Secreted. URL http://www.ncbi.nlm.nih.gov/pmc/articles/PMC3072236/

[11] K. V. Garland, E. Vasquez, C. Pearl, Efficacy of individualized clinical 640 coaching in a virtual reality classroom for increasing teachers' fidelity of implementation of discrete trial teaching, Education and Training in Autism and Developmental Disabilities 47 (December) (2012) 502-515.

URL http://eric.ed.gov/?id=EJ986378

[12] A. Robb, R. Kopper, R. Ambani, F. Qayyum, D. Lind, L. M. Su, B. Lok, 645 Leveraging virtual humans to effectively prepare learners for stressful interpersonal experiences, IEEE Transactions on Visualization and Computer Graphics 19 (4) (2013) 662-670. doi:10.1109/TVCG.2013.35

[13] M. Core, D. Traum, H. C. Lane, W. Swartout, J. Gratch, M. van Lent, a S. Marsella, Teaching Negotiation Skills through Practice and Reflection ${ }_{650}^{6}$ ․ with Virtual Humans, Simulation 82 (11) (2006) 685-701. doi:10.1177/ 0037549706075542 .

URL http://sim.sagepub.com/cgi/doi/10.1177/0037549706075542 
[14] L. Hall, M. Vala, M. Hall, M. Webster, S. Woods, R. Aylett, FearNot's Appearance: Reflecting children's expectations and perspectives, 6th International Conference, IVA, Springer, LNAI 4133,.

URL http://link.springer.com/chapter/10.1007/11821830_33

[15] R. McDonnell, M. Breidt, H. H. Bülthoff, Render me Real? Investi-

a gating the Effect of Render Style on the Perception of Animated Vir-

1. tual Humans, ACM Transactions on Graphics 31 (4) (2012) 1-11. doi: $10.1145 / 2185520.2185587$.

URL http://dl . acm.org/citation. cfm?doid=2185520.2185587

[16] A. von der Pütten, N. Krämer, J. Gratch, How Our Personality Shapes

ם Our Interactions with Virtual Characters-Implications for Research and Development, Intelligent Virtual Agents. URL http: //www . springerlink . com/index/R851090J536U48R0 . pdf

[17] S. Maddock, J. Edge, M. Sanchez, Movement Realism in Computer Facial Animation, 19th British HCI Group Annual Conference, Workshop on Human-animated Characters Interaction 6 (2005) 1-4. http://www.researchgate.net/profile/Steve_Maddock/ publication/228922876_Movement_realism_in_computer_facial_ animation/links/09e4150e6f78d266e7000000.pdf

[18] T. Ni, D. a. Bowman, J. Chen, Increases Display Size and Resolution Improve Task Performance in Information-Rich Virtual Environments, Graphics Interface (2006) 139. URL http://cs.brown.edu/ jchen/publications/2006_GI_IRVE.pdf

[19] K. Johnsen, B. Lok, An Evaluation of Immersive Displays for Virtual Human Experiences, 2008 IEEE Virtual Reality Conference (2008) 133136doi:10.1109/VR.2008.4480764.

II URL http://ieeexplore.ieee.org/lpdocs/epic03/wrapper.htm? arnumber $=4480764$ 
[20] J. N. Bailenson, J. Blascovich, A. C. Beall, J. M. Loomis, Interpersonal Distance in Immersive Virtual Environments, Personality and Social Psychology Bulletin 29 (7) (2003) 819-833. doi:10.1177/0146167203253270

URL http://psp.sagepub.com/content/29/7/819.full.pdf

[23] M. Garau, M. Slater, V. Vinayagamoorthy, a. Brogni, a. Steed, M. Sasse,

a The impact of avatar realism and eye gaze control on perceived quality of

口

[26] A. M. V. D. Pütten, N. C. Krämer, J. Gratch, Who's there? Can a Virtual

[24] J. Rickel, W. Johnson, Task-oriented collaboration with embodied agents in virtual worlds, Embodied conversational agents (2000) 1-29.

URL http://www.isi.edu/isd/VET/eca00.pdf

[25] A. Kotranza, B. Lok, A. Deladisma, C. M. Pugh, D. S. Lind, Mixed reality humans: Evaluating behavior, usability, and acceptability, IEEE Transactions on Visualization and Computer Graphics 15 (3) (2009) 369-382. doi:10.1109/TVCG.2008.195.

URL http://ieeexplore.ieee.org/xpls/abs_all.jsp?arnumber= 4689554 
Agent Really Elicit Social Presence? (2009) 1-7.

URL http://ict.usc.edu/pubs/whosthere.pdf

[27] A. M. Von Der Pütten, N. C. Krämer, J. Gratch, S. H. Kang, "It

a doesn't matter what you are!" Explaining social effects of agents and avatars, Computers in Human Behavior 26 (6) (2010) 1641-1650. doi:10.1016/j.chb.2010.06.012

[28] K. L. Nowak, F. Biocca, The Effect of the Agency and Anthropomorphism on Users' Sense of Telepresence, Copresence, and Social Presence in Virtual Environments, Presence: Teleoperators and Virtual Environments 12 (2003) 481-494. doi:10.1162/105474603322761289.

[29] L. Ring, D. Utami, T. Bickmore, The Right Agent for the Job? The effects of agent visual appearance on task domain, Intelligent Virtual Agents.

URL http://relationalagents . com/publications/render . iva14.pdf

[30] B. Reeves, C. Nass, The Media Equation: How people treat computers, television, and new media like real people and places, CSLI Publications and Cambridge university press, 1996.

[31] C. Nass, K. M. Lee, Does computer-synthesized speech manifest personality? Experimental tests of recognition, similarity-attraction, and consistency-attraction., Journal of experimental psychology. Applied 7 (3) (2001) 171-181. doi:10.1037/1076-898X.7.3.171

[32] E. Lee, C. Nass, S. Brave, Can computer-generated speech have gender?: an experimental test of gender stereotype, CHI'00 extended abstracts on Human factors ...(2000) 289-290doi:http://doi.acm.org/10.1145/ 633292.633461 URL http://dl .acm.org/citation. cfm?id=633461 
[33] C. Nass, B. Fogg, Y. Moon, Can computers be teammates?, International Journal of Human-Computer Studies 45 (1996) 669-678. doi:10.1006/ijhc.1996.0073. URL http://linkinghub.elsevier.com/retrieve/pii/ S1071581996900737

[34] B. Rossen, K. Johnsen, A. Deladisma, S. Lind, B. Lok, Virtual humans elicit skin-tone bias consistent with real-world skin-tone biases, Intelligent Virtual Agentsdoi:10.1007/978-3-540-85483-8\_24. URL http://www . springerlink.com/index/412115740g814803.pdf

${ }_{745}[35]$ D.-P. Pertaub, M. Slater, C. Barker, An experiment on public speaking anxiety in response to three different types of virtual audience, Presence: Teleoperators \& Virtual Environments 11 (1) (2002) 68-78. doi:10.1162/ 105474602317343668

URL http://eprints.ucl.ac.uk/13447/

${ }_{750}[36]$ a. Raij, K. Johnsen, R. Dickerson, B. Lok, M. Cohen, T. Bernard, C. Oxendine, P. Wagner, D. Lind, Interpersonal Scenarios: Virtual Real?, IEEE Virtual Reality Conference (VR 2006) 59-66 doi:10.1109/VR.2006.91.

a URL http://ieeexplore.ieee.org/lpdocs/epic03/wrapper.htm? arnumber $=1667627$

${ }_{755}[37]$ G. M. Lucas, J. Gratch, A. King, L. P. Morency, It's only a computer: Virtual humans increase willingness to disclose, Computers in Human Behavior 37 (2014) 94-100. doi:10.1016/j.chb.2014.04.043

【 URL http://linkinghub.elsevier.com/retrieve/pii/ S0747563214002647

760 [38] D. Kipnis, S. M. Schmidt, I. Wilkinson, Intraorganizational influence tactics: Explorations in getting one's way., Journal of Applied Psychology 65 (4) (1980) 440-452. doi:10.1037/0021-9010.65.4.440.

URL http://content .apa.org/journals/apl/65/4/440 
[39] M. B. Wadsworth, A. L. Blanchard, Influence tactics in virtual teams, Computers in Human Behavior 44 (2015) 386-393. doi:10.1016/j.chb. 2014.11 .026

URL http://dx.doi.org/10.1016/j.chb.2014.11.026

[40] B. Van Knippenberg, H. Steensma, Future interaction expectation and the use of soft and hard influence tactics, Applied Psychology 52 (1) (2003) 55-67. doi:10.1111/1464-0597.00123.

[41] R. C. Ringer, W. R. Boss, Hospital Professionals' Use of Upward Influence Tactics, Journal of Managerial Issues 12 (1) (2000) 92-108. doi:10.1016/0024-3205(88)90461-4.

11 URL http://www.jstor.org/discover/10.2307/40604296?uid= $3739600 \& u i d=2 \& u i d=4 \& u i d=3739256 \&$ sid $=21106635424273$

[42] G. Yukl, C. M. Falbe, Influence tactics and objectives in upward, downward, and lateral influence attempts., Journal of Applied Psychology 75 (2) (1990) 132-140. doi:10.1037/0021-9010.75.2.132.

[43] S. Steizel, E. Rimbau-Gilabert, Upward influence tactics through technology-mediated communication tools, Computers in Human Behavior 29 (2012) 462-472. doi:10.1016/j.chb.2012.04.024.

[44] D. Tjosvold, H. F. Sun, Effects of Influence Tactics and Social Contexts in Conflict: an Experiment on Relationships in China (2001). doi:10.1108/ eb022857.

[45] C. a. Schriesheim, T. R. Hinkin, Influence tactics used by subordinates: A theoretical and empirical analysis and refinement of the Kipnis, Schmidt, and Wilkinson subscales., Journal of Applied Psychology 75 (3) (1990) 246-257. doi:10.1037/0021-9010.75.3.246.

[46] D. Kipnis, S. M. Schmidt, Upward-Influence Styles: Relationship with Performance Evaluations, Salary, and Stress, Administrative Science Quarterly 33 (4) (1988) 528. doi:inf. 
URL http://search.ebscohost.com/login.aspx?direct=true\&db= buh\&AN $=4010204 \&$ site $=$ ehost - live

[47] B. L. Joon Hao Chuah, Andrew Robb, Casey White, Adam Wendling, Samsun Lampotang, Regis Kopper, Exploring Agent Physicality and Social Presence for Medical Team Training, Presence: Teleoperators and Virtual Environments 22 (2) (2013) 141-170.

URL http://www.mitpressjournals.org/doi/abs/10.1162/PRES_a_ 00145\#.VUEF6yFVhBe

800 [48] L. Castillo-Page, Diversity in the Physician Workforce: Facts \& Figures 2010, Association of American Medical Colleges.

a URL https://members.aamc.org/eweb/upload/ DiversityinthePhysicianWorkforceFactsandFigures2010.pdf

[49] T. Bickmore, Relational agents: Effecting change through human-computer relationships, Ph.D. thesis (2003).

URL http://www.ccis.northeastern.edu/home/bickmore/ bickmore-thesis.pdf

[50] L. P. Vardoulakis, L. Ring, B. Barry, C. L. Sidner, T. Bickmore, Designing relational agents as long term social companions for older adults, Intelligent Virtual Agent:doi:10.1007/978-3-642-33197-8-30 URL http://link.springer.com/chapter/10.1007/ 978-3-642-33197-8_30

[51] R. L. Gorden, Basic Interviewing Skills, F. E. Peacock, Itasca, IL, 1998. URL http://books .google.it/books?id=LfoVAAAQBAJ

${ }_{815}[52]$ E. J. Thomas, J. B. Sexton, R. L. Helmreich, Discrepant attitudes about teamwork among critical care nurses and physicians., Critical care medicine 31 (3) (2003) 956-959. doi:10.1097/01.CCM.0000056183.89175.76.

URL http://www.ncbi.nlm.nih.gov/pubmed/12627011 
[53] M. a. Makary, J. B. Sexton, J. a. Freischlag, C. G. Holzmueller, E. A.

820 ㅁ Millman, L. Rowen, P. J. Pronovost, Operating Room Teamwork among Physicians and Nurses: Teamwork in the Eye of the Beholder, Journal of

n the American College of Surgeons 202 (5) (2006) 746-752. doi:10.1016/ j.jamcollsurg.2006.01.017.

URL http://www.ncbi.nlm.nih.gov/pubmed/16648014

825 [54] L. Van Dyne, S. Ang, I. C. Botero, L. V. Dyne, Conceptualizing employee silence and employee voice as multidimensional constructs, Journal of Management Studies 40 (September) (2003) 1359-1392. doi:10.1111/1467-6486.00384.

URL http://onlinelibrary.wiley.com/doi/10.1111/1467-6486. 


\title{
Acknowledgements

The authors would like to thank Theresa Hughes, Terry Sullivan, and David Lizdas for their help in developing the Speaking Up exercise and recruiting participants, as well as the nurses who agreed to

\begin{abstract}
participate in this study. This work was supported in part by NSF Grant 1161491.
\end{abstract}

